Published in: Oehler, J. (2010) (ed.): Der Mensch - Evolution, Natur und Kultur: Beiträge zu unserem heutigen Menschenbild. Berlin: Springer, 261-283.

\title{
Der evolutionäre Naturalismus in der Ethik
}

\author{
von Marie I. Kaiser
}

Charles Darwin hat eindrucksvoll gezeigt, dass der Mensch ebenso wie alle anderen Lebewesen ein Produkt der biologischen Evolution ist. Die sich an Darwin anschließende Forschung hat außerdem plausibel gemacht, dass sich nicht nur viele der körperlichen Merkmale des Menschen, sondern auch (zumindest einige) seiner Verhaltensdispositionen in adaptiven Selektionsprozessen herausgebildet haben. Die Vorstellung, dass auch die menschliche Moralität evolutionär bedingt ist, scheint daher auf den ersten Blick ganz überzeugend. Schließlich hat die Evolutionstheorie in den vergangenen Jahrzehnten in vielen Bereichen (auch außerhalb der Biologie) ihre weitreichende Bedeutung unter Beweis gestellt. Warum sollte, so könnte man beispielsweise fragen, gerade die Fähigkeit des Menschen, moralische Normen aufzustellen und gemäß ihnen zu handeln, nicht evolutionär erklärt werden können? Und warum sollte eine solche evolutionäre Erklärung der menschlichen Moralität irrelevant für die Rechtfertigung moralischer Normen sein? Warum sollte die Ethik eine Bastion der Philosophen bleiben, für die evolutionsbiologische Forschungsergebnisse über den Menschen und seine nächsten Verwandten keinerlei Relevanz besitzen?

Dass eine Verbindung von Evolution und Moral weitaus komplizierter und kontroverser ist, als hier angedeutet, davon zeugt schon die lange andauernde und zum Teil sehr hitzig geführte Debatte über die Möglichkeiten und Grenzen einer evolutionären Naturalisierung der Ethik. Seit und mit Darwin haben zahlreiche Philosophen und Biologen versucht, aus der Darwinschen Evolutionstheorie Konsequenzen für die Ethik abzuleiten. In seiner extremsten Form wird das Projekt der Naturalisierung der Ethik zum Versuch einer feindlichen Übernahme der philosophischen Ethik durch die Biologen, wie die folgende Forderung des Begründers der Soziobiologie, Edward O. Wilson, zeigt: „[T] he time has come for ethics to be removed temporarily from the hands of the philosophers and biologicized.“ (1975: 562) Die Philosophen lassen sich die Ethik jedoch nicht so leicht aus den Händen nehmen und entgegnen: Das gewagte Projekt der evolutionären Ethiker, die Ableitung moralischer Normen aus evolutionsbiologischen Tatsachen, sei doch schon seit David Hume als auf einem Sein-Sollens-Feblschluss (Treatise: Buch III, Teil I) basierend entlarvt worden. Alle übrigen Versuche einer Verbindung von Evolution und Moral, wie etwa die Erklärung des menschlichen Moralverhaltens auf Basis der Evolutionstheorie, seien trivial und aufgrund ihrer „evaluativen Irrelevan ¿“ (Bayertz 1993a: 165) schlichtweg uninteressant.

Mit Philip Kitcher (1993) möchte ich betonen, dass immer noch viele Uneinigkeiten in der Debatte darauf zurückzuführen sind, dass die verschiedenen Projekte einer Verbindung von Evolution und Moral und die damit verbundenen Begrifflichkeiten und Argumentationsstrategien nicht hinreichend klar analysiert und differenziert werden. Das wesentliche Ziel meines Aufsatzes besteht deshalb darin, mehr analytische Schärfe in die Debatte um den evolutionären Naturalismus in der Ethik zu bringen. Im Fokus meiner Untersuchung wird die Frage stehen, worin genau das Projekt einer evolutionären Naturalisierung der Ethik besteht und in welchem Sinne dabei von ,Naturalismus' die Rede ist. Um diese Frage zu beantworten, werde ich in zwei Hauptschritten vorgehen: Im Anschluss an die noch sehr allgemeine Feststellung, dass Naturalisten den Naturwissenschaften eine große Bedeutung zuschreiben (Abschnitt 1), werde ich zunächst die allgemeine Debatte um den Naturalismus betrachten und analysieren, in welchem Sinne man ein Naturalist sein kann (Abschnitt 2). Im Zuge dieser Untersuchung wird sich herausstellen, dass man drei verschiedene 
Naturalismusbegriffe unterscheiden muss: den ontologischen (Abschnitt 2.1), den (schwachen bzw. starken) methodologischen (Abschnitt 2.2) und den empirischen Naturalismus (Abschnitt 2.3). Die im ersten Teil gewonnenen Ergebnisse werde ich im zweiten Schritt auf den speziellen Fall des evolutionären Naturalismus in der Ethik anwenden (Abschnitt 3). Entsprechend den drei klassischen Bereichen der Ethik - der Metaethik (Abschnitt 3.1), der normativen Ethik (Abschnitt 3.2) und der deskriptiven Ethik (Abschnitt 3.3) - werde ich drei Typen von Projekten einer evolutionären Naturalisierung der Ethik unterscheiden, sie in ihren Grundzügen erläutern und deutlich machen, um welche Version des Naturalismus es sich jeweils handelt.

\section{Der Naturalismus und die vermeintlich große Bedeutung der Naturwissen- schaften}

Der Ausdruck ,Naturalismus ist innerhalb der vergangenen Jahrzehnte ein Sammelbegriff für ganz unterschiedliche philosophische Positionen geworden. Er spielt innerhalb der Erkenntnistheorie, der Philosophie des Geistes, der Wissenschaftstheorie, der Religionsphilosophie, der Metaphysik und auch innerhalb der Ethik eine bedeutende Rolle. Eine präzise Definition des Ausdrucks findet man allerdings nur selten. Viele Autoren konzentrieren sich stattdessen auf die Aufzählung und kritische Diskussion der einzelnen als naturalistisch bezeichneten Positionen (Papineau 2009, Ritchie 2008).

Versucht man trotz der Bedeutungsvielfalt des Begriffes einige zentrale Charakteristika naturalistischer Positionen zu identifizieren, stößt man zunächst auf folgenden Punkt: Im Gegensatz zu klassischen Positionen wie z. B. dem ethischen Naturalismus, der zu den ältesten philosophischen Deutungsprogrammen der Moral gehört, ist für den modernen Naturalismus (seit Ende des 19. Jahrhunderts) nicht der Begriff der Natur, sondern der Begriff der Naturwissenschaft zentral. Dieses Charakteristikum des modernen Naturalismus ist das Ergebnis der Verfallsgeschichte des „qualitativen Naturbegriffs“, an deren Ende die Natur nur noch als dasjenige angesehen wird, das „Gegenstand einer empirischen (Gesetzes-)Wissenschaft ist“ (Mittelstraß 1984: 962.) Entsprechend dieser Fokussierung ist für die modernen Naturalisten typisch, dass sie den Naturwissenschaften eine besondere Wertschätzung entgegenbringen, d. h. die Bedeutung der Naturwissenschaften im Vergleich zu und für andere Disziplinen als sehr hoch einstufen. Aufgrund des großen Erfolges der Naturwissenschaften bei der Erforschung und Erklärung der Welt sollten - so ihre Argumentation - auch die Philosophen endlich anerkennen, welche enorme Bedeutung die Naturwissenschaft für die Philosophie habe. Ein evolutionärer Naturalist in der Ethik behauptet dementsprechend, dass eine bestimmte Naturwissenschaft, nämlich die biologische Evolutionstheorie, von großer Bedeutung für einen bestimmten Teil der Philosophie, nämlich für die Ethik, ist.

Was genau ist jedoch mit der großen Bedeutung der Evolutionstheorie für die Ethik gemeint und welche Konsequenzen ergeben sich aus ihr? Geht es um die These, dass die Ethik bei genauerer Betrachtung letztlich nichts anderes als Evolutionstheorie sein sollte und aus diesem Grund durch sie ersetzt werden sollte? Oder besteht die Bedeutung der Evolutionstheorie vielmehr darin, dass sie spezifische Methoden bereit stellt, die zur Beantwortung ethischer Fragestellungen herangezogen werden sollen, bzw. dass sie bestimmte empirische Daten liefert, die in die ethische Argumentation einbezogen werden sollen? Oder geht es lediglich darum, die Fortschritte anzuerkennen, die Evolutionstheoretiker in den vergangenen Jahrzehnten bei der Erforschung der menschlichen Moralität gemacht haben? Letzteres kann sofort verneint werden. Will der Naturalismus in der heutigen Zeit eine substantielle, interessante philosophische Position sein, so muss er über eine bloße Solidaritäts- oder Respektbekundung gegenüber den Naturwissenschaften hinausgehen. Ebenso lässt auch die vage These, dass in der Welt alles mit rechten 
Dingen zugeht, und die darin enthaltene Ablehnung jeglicher Art von Wunderglauben, Obskurantismus und Okkultismus den Naturalismus noch nicht zu einer substantiellen philosophischen Position werden. Das Ziel der Bestimmung des Begriffs ,Naturalismus' kann nicht darin bestehen, die Bedeutung des Begriffs so vage und allgemein zu lassen, dass man wie Roy Wood Sellars zu dem Schluss kommt: „[W]e are all naturalists now.“ (Sellars 1922: i).

Wenn der evolutionäre Naturalismus in der Ethik nicht bloß darin bestehen kann, den bisherigen Erfolg der Evolutionstheorie anzuerkennen, worin genau besteht dann aber die vermeintlich große Bedeutung der Evolutionstheorie für die Ethik und in welchem Sinne spricht man von der Naturalisierung der Moralphilosophie? Um diese Fragen zu beantworten, ist es nützlich, zunächst einen Blick in die allgemeine Debatte um den Naturalismus zu werfen und zu untersuchen, welche verschiedenen Typen von Naturalisierungsprojekten man unterscheiden kann.

\section{$2 \quad$ In welchem Sinne kann man Naturalist sein?}

\subsection{Der ontologische Naturalismus}

In der Debatte werden üblicherweise zwei Varianten des Naturalismus unterschieden: der ontologische und der methodologische. Der ontologische Naturalismus ist eine These darüber, welche Entitäten in der Welt existieren. Ein ontologischer Naturalist behauptet, dass nur natürliche Entitäten, nicht aber nicht-natürliche Entitäten (wie z. B. Gott, Hexen, Schöpfungsakte, moralische Eigenschaften etc.) existieren. Für diese Position ist auch der Spruch „Alles ist Natur." charakteristisch. Vermeintliche nicht-natürliche Entitäten existierten nur, insofern sie auf natürliche Entitäten reduzierbar seien. In den Worten von J. J. C. Smart: Ontologische Naturalisten sind der Ansicht, dass in der Welt nichts über natürliche Entitäten Hinausgehendes existiert („,nothing over and above" Smart 1959: 142). Die Erkenntnis, dass vermeintlich nicht-natürliche Entitäten auf natürliche Entitäten reduzierbar sind, kann einerseits zur Folge haben, dass die nichtnatürlichen Entitäten eliminiert werden. So spricht man z. B. heutzutage nicht mehr in einer Weise von Hexen, als ob sie existierten. Andererseits kann die Folge aber auch sein, dass die vermeintlich nicht-natürlichen Entitäten erbalten bleiben, aber gezeigt wird, dass sie nichts anderes als natürliche Entitäten sind (Kim 2006: 276). Man kann als ontologischer Naturalist in der Ethik z. B. auch weiterhin davon sprechen, dass bestimmten Handlungen die moralische Eigenschaft zukommt, moralisch wertvoll zu sein, wenn klar bleibt, dass moralische Eigenschaften als solche nicht existieren, sondern dass sie auf natürliche Eigenschaften reduziert werden können.

Ähnlich wie beim Physikalismus die drängende Frage offen bleibt, was mit dem Physikalischen eigentlich genauer gemeint ist, muss auch ein ontologischer Naturalist näher bestimmen, was er unter dem Natürlichen versteht. Wie im vorherigen Abschnitt bereits deutlich wurde, zeichnet sich der moderne Naturalismus dadurch aus, dass in ihm der Begriff der Naturwissenschaften zentral ist und dementsprechend ,natürlich' in der Regel mit ,naturwissenschaftlich erforschbar ${ }^{6}$ bzw. ,Gegenstand der Naturwissenschaften sein ${ }^{`}$ ausbuchstabiert wird (z. B. Armstrong 1983: 82). Die Naturwissenschaften - ihre Forschungspraxis (Cartwright 1999) oder ihre erfolgreichsten und am besten bestätigten Theorien (Esfeld 2007) - werden dadurch als alleinige Quelle der Ontologie ausgezeichnet: Was in der Welt existiert, darüber können ausschließlich die Naturwissenschaften Auskunft geben. ${ }^{1}$ Wilfried Sellars formuliert diese These wie folgt: „,...] in the dimension of describing and explaining the world, science is the measure of all things, of

\footnotetext{
1 Viele der ontologischen Naturalisten vertreten die Auffassung, dass zu den erfolgreichsten und am besten bestätigten Theorien vor allem oder ausschließlich die Theorien der Physik gehören. Der ontologische Naturalismus fällt in diesen Fällen mit einem (ontologischen) Physikalismus zusammen (Maudlin 2007).
} 
what is that it is, and of what is not that it is not." (1956: 173) Aus dieser These folgt allerdings nicht, dass es auch Naturwissenschaftler sind, denen die Aufgabe zufällt, Ontologie zu betreiben. Einige Autoren sind der Meinung, dass die Naturwissenschaftler lediglich das Material liefern (die wissenschaftliche Praxis bzw. die Theorien), aus dem die Philosophen dann die ontologischen Thesen ableiten (Ladyman u. Ross 2007).

Gegen eine solche Vorgehensweise kann zu Recht eingewandt werden, dass die wissenschaftliche Praxis bzw. die wissenschaftlichen Theorien die ontologischen Thesen, die einige Philosophen aus ihnen herauszulesen meinen, gar nicht hergeben. Denn große Teile der Naturwissenschaften - ihre Praxis ebenso wie ihre Theorien - sind meiner Ansicht nach ontologisch unterbestimmt, d. h., sie sind mit alternativen Ontologien gleichermaßen vereinbar. Philosophen, die Metaphysik der Naturwissenschaften betreiben, begehen zu häufig den Fehler, ontologische Thesen aus den Naturwissenschaften abzuleiten, die sie eigentlich selbst in sie hineingelegt haben. Eine solche Position kann nur als pseudo-naturalistisch bezeichnet werden, insofern ihre Vertreter vorgeben, die Naturwissenschaften als alleinige Quelle ihrer Ontologie zu nutzen, dies aber de facto nicht tun. Einige ontologische Naturalisten wie z. B. Arthur Fine mahnen aus ähnlichen Gründen zur Vorsicht und fordern, dass der ontologische Naturalismus mit einer natürlichen ontologischen Einstellung (ㅁatural ontological attitude [NOA]) verbunden sein solle: ,[T]ry to take science on its own terms, and try not to read things into science." (Fine 1996: 149)

\subsection{Der methodologische Naturalismus}

Durch die Gleichsetzung des Natürlichen mit dem naturwissenschaftlich Erforschbaren entsteht eine enge Verbindung des ontologischen zum methodologischen Naturalismus. In einer ersten Annäherung ist der methodologische Naturalismus eine Antwort auf die Frage, worin die erfolgreichste Weise, die Welt zu erforschen und Wissen über sie zu erlangen, besteht - nämlich in der Anwendung naturwissenschaftlicher Methoden - und was daraus für das Verhältnis zwischen der Philosophie und den Naturwissenschaften folgt. Diese Begriffsbestimmung ist deshalb noch recht vage, weil unter dem Etikett ,methodologischer Naturalismus' ganz verschiedene Fragestellungen diskutiert werden und Naturalisten auf einige dieser Fragen unterschiedliche Antworten geben. Im Folgenden werde ich diese verschiedenen Fragestellungen und Thesen unterscheiden und wichtige Beziehungen zwischen ihnen erläutern. Auf diese Weise werden wir der Antwort auf die Frage näher kommen, was ein methodologischer Naturalist behauptet.

\subsubsection{Die schwache und starke Variante des methodologischen Naturalismus}

Vor allem in Debatten um das Verhältnis zwischen Naturwissenschaft und Religion ist mit der Position des methodologischen Naturalismus häufig eine These darüber verbunden, wie man die Phänomene der natürlichen Welt erforschen sollte.

\section{Schwacher methodologischer Naturalismus:}

Es sollte versucht werden, alle Phänomene unter Anwendung ausschließlich naturwissenschaftlicher Methoden zu erforschen.

Der methodologische Naturalismus in seiner schwachen Form ist eine methodologische Empfehlung bəw. Vorschrift, wie man bei der Erforschung der Phänomene der Welt vorgehen sollte nämlich indem man ausschließlich naturwissenschaftliche Methoden anwendet. Phänomene sollten erklärt und Hypothesen sollen getestet werden, indem man sich ausschließlich auf natürliche Entitäten, nicht aber auf okkulte Kräfte oder übernatürliche Wesen bezieht. Mit dieser Variante des Naturalismus als einem Forschungsprogramm ist vereinbar, dass es Phänomene gibt, bei 
denen die vorgeschriebene Strategie fehlschlägt, weil diese Phänomene prinzipiell nicht naturwissenschaftlich erforschbar und erklärbar sind. Der schwache methodologische Naturalismus lässt auf diese Weise z. B. Raum für einen Schöpfungsglauben und ermöglicht eine Kombination von Evolutionstheorie und Religion (Kummer 2009, Ruse 2008). Im Extremfall kann der schwache methodologische Naturalismus zu der These abgeschwächt werden, dass lediglich die Phänomene, die in den Gegenstandsbereich der Naturwissenschaften fallen, mit ausschließlich naturwissenschaftlichen Methoden erforscht werden sollten (Forrest 2000, Kurtz 1998). Ich möchte mich allerdings der Meinung von Keil und Schnädelbach anschließen, die behaupten, dass der Naturalismus erst dann spannend wird, wenn er die Anwendbarkeit der naturwissenschaftlichen Methode in Bereichen außerbalb der Naturwissenschaften postuliert (Keil u. Schnädelbach 2000: 20).

Nach Ansicht einiger Naturalisten sowie Naturalismus-Kritiker geht der schwache methodologische Naturalismus (auch in seiner stärkeren Form) nicht weit genug. Sie charakterisieren den methodologischen Naturalismus stattdessen als eine Verbindung zweier stärkerer Thesen:

\section{Starker methodologischer Naturalismus:}

Die Methoden der Naturwissenschaften (1) besitzen einen einzigartigen Status und (2) sind universell.

(1) Einzigartiger Status der naturwissenschaftlichen Methoden:

Die Methoden der Naturwissenschaft sind erfolgreich und der einzige verlässliche Weg zur Wahrheit. Aus diesem Grund besitzen die Naturwissenschaften ein Erklärungsprivileg.

Diese erste These des starken methodologischen Naturalisten wird auch unter dem Stichwort ,Mittelbeschränkung` erörtert (Vollmer 2000: 48-50). Dadurch, dass der Naturalist die Methoden der Naturwissenschaften als den einzigen verlässlichen Weg zur Wahrheit auszeichnet, beschränkt er die Mittel, die zur Erforschung und Erklärung der Welt zugelassen sind. Der starke methodologische Naturalist ist mit dieser These allerdings nicht auf einen Infallibilismus verpflichtet, $\mathrm{d}$. $\mathrm{h}$. auf die These, dass die naturwissenschaftlichen Methoden immer zur Wahrheit führen müssen. Der bisherige Erfolg der Naturwissenschaften wird meist als wichtigster Beleg für die Güte und Verlässlichkeit ihrer Methoden angeführt. Diese Strategie kann aber nicht darüber hinwegtäuschen, dass die Behauptung, die naturwissenschaftlichen Methoden seien der einzige verlässliche Weg zur Wahrheit, sehr stark ist und gewichtiger philosophischer Argumente bedarf. Viele der Naturalisten wehren sich jedoch schon gegen die Voraussetzung einer solchen Argumentation, nämlich gegen die Spezifizierung des Begriffs der naturwissenschaftlichen Methoden. Um den Naturwissenschaften nicht vorzugreifen, sie nicht zu bevormunden und neutral gegenüber jeder Wissenschaftsklassifikation zu bleiben, geben viele Naturalisten explizit nicht an, wodurch genau die naturwissenschaftlichen Methoden charakterisiert sind, sondern erklären ganz nach dem Motto „Wherever science will lead, I will follow.“ (Keil u. Schnädelbach 2000: 22) ihre Solidarität mit dem Gang der Naturwissenschaften selbst. So bequem eine solche neutrale Haltung auch sein mag, sie lässt den starken methodologischen Naturalismus (wie auch den schwachen) konturlos und damit unattraktiv werden. Jemand, der den Naturwissenschaften aufgrund ihrer Methoden einen einzigartigen epistemischen Status zuschreibt, sollte etwas darüber sagen können, was diese Methoden sind und wie sie sich von den Methoden anderer Wissenschaften (und NichtWissenschaften) unterscheiden. Eine solche Spezifizierung des Begriffs muss auch nicht statisch sein, sondern kann gegenüber Veränderungen der in der wissenschaftlichen Praxis etablierten Methoden offen bleiben. Erst auf der Basis einer solchen Spezifizierung des Begriffs der naturwissenschaftlichen Methoden kann ein methodologischer Naturalist im starken Sinne seine 
Behauptung begründen, die Methoden der Naturwissenschaft seien der einґige verlässliche Weg zur Wahrheit.

Die Plausibilität der zweiten These des starken methodologischen Naturalisten setzt die Plausibilität der soeben erläuterten ersten These voraus:

(2) Universalität der naturwissenschaftlichen Methoden (globale Version):

Aufgrund ihres einzigartigen Status ist es möglich und erfolgsversprechend, die Methoden der Naturwissenschaft universell (d. h. in Bezug auf alle Phänomenbereiche und alle Fragestellungen) anzuwenden.

Hat man einmal nachgewiesen, dass die Methoden der Naturwissenschaften der einzige verlässliche Weg zur Wahrheit sind, erscheint es nach Ansicht des starken methodologischen Naturalisten plausibel, den Anwendungsbereich dieser Methoden auf die Fragestellungen und Phänomene anderer Wissenschaften (und Nicht-Wissenschaften) und letztlich auf die gesamte Welt auszudehnen. An dieser These wird der Unterschied zur schwachen Variante des methodologischen Naturalismus besonders deutlich: Starke methodologische Naturalisten behaupten nicht nur, dass versucht werden sollte, die naturwissenschaftlichen Methoden zur Erforschung aller Phänomene und zur Beantwortung aller Fragestellungen anzuwenden, sondern dass diese Anwendung außerdem in Bezug auf alle Phänomene und Fragen möglich und vor allem erfolgsversprechend (bzw. erfolgreich) ist. Nach Auffassung von Keil und Schnädelbach ist dieser universale Anspruch keine optionale Zutat des methodologischen Naturalismus, sondern es liegt ,in der Logik des Naturalismus [...], keine Enklaven zu dulden.“ (Keil u. Schnädelbach 2000: 20 f.)

Aus dem universalen Anspruch folgt jedoch nicht, dass es nicht möglich ist, einen starken methodologischen Naturalismus zu vertreten, der lediglich lokal ist, d. h. in dem die Anwendbarkeit und erfolgsversprechende Anwendung naturwissenschaftlicher Methoden (außerhalb der Naturwissenschaften) nur für eine oder mehrere spezifische Disziplinen behauptet wird. Naturalistische Positionen werden meist in dieser lokalen Variante verteidigt. So vertritt Willard V. O. Quine einen Naturalismus in Bezug auf die Erkenntnistheorie (Quine 1975), Kitcher in Bezug auf die Ethik (Kitcher 1993, 2006a,b) und Eckard Voland in Bezug auf die Religion (Voland 2009) - um nur einige lokale Naturalisten zu nennen.

(2) Universalität der naturwissenschaftlichen Methoden (lokale Version):

Aufgrund ihres einzigartigen Status ist es möglich und erfolgsversprechend, die Methoden der Naturwissenschaft in Bezug auf die Phänomene und Fragestellungen des nichtnaturwissenschaftlichen Bereiches $\mathrm{X}$ anzuwenden.

Befürworter einer lokalen Variante des starken methodologischen Naturalismus sind meist nicht der Meinung, dass die Anwendbarkeit und erfolgsversprechende Anwendung naturwissenschaftlicher Methoden (außerhalb der Naturwissenschaften) auf diese Disziplinen beschränkt ist. Die meisten lokalen Naturalisten verhalten sich lediglich agnostisch gegenüber einer Ausweitung ihrer Thesen auf andere bzw. auf alle Gegenstandsbereiche und Fragestellungen.

\subsubsection{Das Verhältnis zwischen den Naturwissenschaften und der Philosophie}

Die Debatte um den Naturalismus wird unter Philosophen besonders hitzig geführt, wenn es um die Anwendung naturwissenschaftlicher Methoden auf ihre eigene Disziplin geht. Welches Bild vom Verhältnis zwischen den Naturwissenschaften und der Philosophie ist aber mit den beiden Thesen des starken methodologischen Naturalismus verbunden? Ich möchte im Folgenden zpei Aspekte dieses Bildes unterscheiden, die in der Naturalismus-Debatte meiner 
Ansicht nach nicht klar genug voneinander getrennt werden: Zum einen kann man die Frage stellen, wie viel Naturwissenschaft nach Ansicht der Naturalisten in der Philosophie enthalten sein sollte und zum anderen kann man sich fragen, wie viel Philosophie nach Meinung der Naturalisten in der Naturwissenschaft stecken sollte. In Bezug auf die erste Frage herrscht unter den methodologischen Naturalisten eine starke Uneinigkeit, wohingegen sie sich in der Beantwortung der zweiten Frage weitgehend einig sind.

Kommen wir zur ersten Frage: Wie viel Naturwissenschaft sollte - nach Meinung der Naturalisten - in der Pbilosophie (bzw. in spezifischen Disziplinen der Philosophie) stecken? Sie zielt auf diejenige Frage ab, die im Zentrum dieses Artikels steht: Was versteht man unter der Naturalisierung der Philosophie (bzw. der Ethik) und wie weit kann sie gehen? Sind die beiden Thesen des starken methodologischen Naturalisten korrekt, so folgt daraus, dass auch in der Philosophie der ein₹ige verlässliche Weg zur Wahrheit in der Anwendung naturwissenschaftlicher Methoden besteht. Diese Behauptung scheint auf den ersten Blick seltsam, sind die Naturwissenschaften doch allesamt empirische Disziplinen, in denen es um das Erheben und Systematisieren empirischer Daten über die Welt geht. Die Philosophie scheint hingegen eher durch nicht-empirische Methoden wie Begriffsanalyse und rationales Argumentieren charakterisiert zu sein - wie kann also jemals Philosophie mit naturwissenschaftlichen Methoden betrieben werden? Methodologische Naturalisten geben verschiedene Antworten auf diese Frage, von denen ich die zwei wichtigsten hier kurz skizzieren werde.

Die erste Strategie, für eine methodologische Naturalisierung der Philosophie zu argumentieren, besteht darin, auf die Identität bzw. die Kontinuität hinzuweisen, die nach Meinung einiger Naturalisten in methodischer Hinsicht zwischen der Philosophie und den Naturwissenschaften besteht. In der Philosophie würden ausschließlich Methoden verwendet (wie z. B. die Konstruktion synthetischer Theorien über die Welt, die Durchführung von (Gedanken-) Experimenten etc.), die auf einer allgemeinen Ebene betrachtet identisch mit den Methoden der Naturwissenschaften seien bzw. die nicht klar von diesen abgegrenzt werden könnten. Es gebe folglich keine genuin philosophischen Methoden und - wenn überhaupt - nur graduelle, aber keine prinzipiellen methodischen Unterschiede zwischen der Philosophie und den Naturwissenschaften. Die Verschiedenartigkeit der beiden Disziplinen komme lediglich dadurch zustande, dass in ihnen unterschiedliche Fragestellungen und Ziele verfolgt, unterschiedliche Phänomene erforscht und unterschiedliche Begriffe und Prinzipien formuliert würden (Papineau 2009: 12-19). Gemäß dieser Argumentationsstrategie ist die Philosophie also insofern naturalisiert, als genuin philosophische Fragestellungen unter Anwendung ausschließlich naturwissenschaftlichen Methoden beantwortet werden. Insofern sich diese These auf die derzeit vorbandene Philosophie bezieht, liefern die methodologischen Naturalisten nur eine Neubeschreibung bzw. -charakterisierung der vorhandenen philosophischen Methoden und ihres Verhältnisses zu den naturwissenschaftlichen Methoden (Koppelberg 1996, 2000). Bezieht sich die These der Naturalisten hingegen nicht auf die vorhandene, sondern auf die in einem noch näher zu spezifizierenden Sinne, richtige 'Philosophie, bekommt sie einen normativen Charakter. Die Identität bzw. Kontinuität wird nicht zwischen den Methoden der Naturwissenschaften und allen in der Philosophie tatsächlich verwendeten Methoden behauptet, sondern zwischen den Methoden der Naturwissenschaften und den Methoden der ,richtigen' Philosophie, die allerdings erst noch (durch Elimination und Hinzufügen bestimmter Methoden) hergestellt werden muss. Eine derartige Forderung steht auch im Fokus der zweiten Verteidigungsstrategie einer methodologischen Naturalisierung der Philosophie.

Laut dieser zweiten Strategie besitze die Philosophie zwar genuin philosophische Methoden. Diese seien aber aufgrund ihres nicht-naturwissenschaftlichen Charakters kein verlässlicher Weg 
zur Wahrheit und sollten daher aus der Philosophie verbannt werden. Das Resultat sei eine neue Philosophie, die ihren Platz innerbalb der Naturwissenschaften habe. Im Zentrum der naturalistischen Kritik steht neben einem reinen Apriorismus auch die so genannte Lehnstublphilosophie, deren Vertreter in ihrer philosophischen Argumentation höchstens solche empirischen (common sense)-Fakten berücksichtigen, die vom Lehnstuhl aus gewusst werden können. Quine, der bekannteste Befürworter dieses Naturalisierungsprojektes für die Erkenntnistheorie, formuliert seine Vorstellung wie folgt:

„[]ch meine, [...] daß die Erkenntnistheorie auch weiterhin fortbesteht, jedoch in einem neuen Rahmen und mit einem geklärten Status. Die Erkenntnistheorie oder etwas Ähnliches erhält ihren Platz innerhalb der Psychologie und somit innerhalb der empirischen Wissenschaften.“ (Quine 1975: 114 f.)

Quines Idee ist allerdings nicht, dass die traditionellen erkenntnistheoretischen Fragestellungen erhalten bleiben und lediglich versucht werden soll, diese Fragen durch Anwendung naturwissenschaftlicher Methoden zu beantworten. Vielmehr solle die traditionelle Erkenntnistheorie vollständig durch Naturwissenschaften wie die Kognitionswissenschaften ersetəిt werden. Aus diesem Grund wird Quines Variante des starken methodologischen Naturalismus auch als „replacement naturalism“ (Feldman 2008: 2) bezeichnet. Im Gegensatz zur ersten Argumentationsstrategie besteht nach Ansicht von Naturalisten wie Quine also nicht nur zwischen den Methoden der Naturwissenschaften und der ,richtigen' Philosophie eine Identität bzw. Kontinuität, sondern auch zwischen ihren Gegenstandsbereichen und Fragestellungen. Eine derartige Naturalisierung der Philosophie ist nur möglich, wenn man zuvor das Verständnis von Philosophie verändert, indem man den ,eigentlichen' bzw. ,richtigen' Gegenstandsbereich und die ,eigentlichen' bzw. ,richtigen Fragestellungen der Philosophie identifiziert. Erst auf der Basis eines solch veränderten Philosophieverständnisses kann ein Naturalist wie Quine behaupten, dass die gesamte Philosophie (bzw. Erkenntnistheorie) unter Anwendung ausschließlich naturwissenschaftlicher Methoden betrieben werden kann. Die replacement-Version des starken methodologischen Naturalismus wird aufgrund seines fragwürdigen Philosophieverständnisses und zahlreichen anderen Problemen heutzutage von nur wenigen Autoren vertreten (Kornblith 1994, Stich 1993). ${ }^{2}$

Bei der Beantwortung der zweite Frage, wie viel Philosophie in der Naturwissenschaften stecken sollte, sind sich die methodologischen Naturalisten einig. Ihre einhellige Antwort lautet: überhaupt keine! Sie stellen sogar ein Einmischungsverbot für Philosophen in die Naturwissenschaften auf. Es richtet sich nicht nur gegen Wissenschaftstheoretiker, die versuchen, die eine naturwissenschaftliche Methode zu bestimmen, ohne dabei mehr als einen Blick in die faktische Wissenschaftspraxis zu werfen, der zeigen würde, wie methodisch vielfältig die Naturwissenschaften sind. ${ }^{3}$ Das Verbot richtet sich gegen jeden Versuch einer Grundlegung der Naturwissenschaften

\footnotetext{
${ }^{2}$ Schon Jaegwon Kim hat an Quines naturalisiertem Verständnis von Erkenntnistheorie kritisiert, dass die zentralen Fragen der traditionellen Erkenntnistheorie unbeantwortet bleiben (Kim 1988: 390). Ein weiteres wichtiges Problem besteht darin, dass die These des methodologischen Naturalismus selbst keine naturwissenschaftliche Aussage ist, sondern eine philosophische These darstellt, die nicht durch Anwendung naturwissenschaftlicher Methoden begründet werden kann (Hartmann u. Lange 2000: 151-158).

${ }^{3}$ Innerhalb der Wissenschaftstheorie vertreten Naturalisten folglich eine rein deskriptive Wissenschaftstheorie und lehnen jede Form von normativer Wissenschaftstheorie ab. Die Aufgabe der Philosophen sei es, die verschiedenen Elemente der vorhandenen Wissenschaftspraxis adäquat zu beschreiben, d. h. sie lediglich zu rekonstruieren und zu erklären, nicht aber den Wissenschaftlern Vorscbriften darüber zu machen, wie sie zu forschen hätten. Dieser Trend hin zu einer sehr nah an der tatsächlichen Forschungspraxis der Wissenschaftler orientierten Wissenschaftstheorie ist vor allem im angelsächsischen Raum weit verbreitet und ist meiner Ansicht nach für die Wissenschaftstheorie äußerst gewinnbringend (Carrier 2007, Gesang 2005).
} 
durch eine Erste Philosophie (Quine 1980: 21). Methodologische Naturalisten vertreten eine „Antifundierungsthese“ (Koppelberg 2000: 83): Kein Philosoph habe bisher überzeugend begründen können, dass und warum die Philosophie gegenüber den Naturwissenschaften einen epistemisch privilegierten Status einnehme. Aus diesem Grund könne die Philosophie auch nicht das Fundament der Naturwissenschaften bereitstellen. Fragen wie, Wie betreibt man erfolgreich Wissenschaft?‘ und ,Worüber kann man auf welche Weise Wissen erlangen?‘ könnten nicht Philosophen, sondern nur die Naturwissenschaftler selbst beantworten. Die Rechtfertigung des Einmischungsverbotes ist unabhängig davon, ob die methodologischen Naturalisten überzeugend für den privilegierten Status und die Universalität naturwissenschaftlicher Methoden argumentieren können oder nicht.

\subsection{Der empirische Naturalismus}

In der Debatte um den methodologischen Naturalismus und seine vermeintlichen Konsequenzen für die Philosophie taucht manchmal eine etwas andersgeartete Version des Naturalismus auf, in der es nicht darum geht, dass die Philosophie ausschließlich mit naturwissenschaftlichen Methoden betrieben werden sollte. Stattdessen geht es um die Forderung, dass Philosophen in ihrer Argumentation das empirische Wissen der Naturwissenschaften berücksichtigen sollten, weil es für die Beantwortung vieler philosophischer Fragestellungen einschlägig und unverzichtbar sei. Diese Version des Naturalismus wird meist auch unter der Rubrik ,methodologischer Naturalismus' diskutiert und mit den zuvor dargestellten Positionen des schwachen und starken methodologischen Naturalismus vermischt. Ich halte diese Vermischung für einen großen Missstand in der Debatte und möchte daher für diese Variante des Naturalismus die neue Bezeichnung ,empirischer Naturalismus‘ einführen. Empirische Naturalisten sind nicht der Meinung, dass die Philosophie (bzw. eine spezifische Disziplin der Philosophie) dadurch naturalisiert werden soll, dass genuin philosophische Fragestellungen ausschließlich mit naturwissenschaftlichen Methoden beantwortet werden sollen oder die Philosophie vollständig durch die Naturwissenschaften ersetzt werden soll. Stattdessen verstehen empirische Naturalisten unter einer naturalisierten Philosophie eine Philosophie, deren Vertreter in ihre Beantwortung genuin philosophischer Fragen unter Anwendung genuin philosophischer Methoden relevante empirische Erkenntnisse, die Naturwissenschaftler im Rahmen der Beantwortung ihrer eigenen Fragen mit ihren eigenen Methoden erlangt haben, mit einberiehen.

Einige Autoren wie Koppelberg sprechen auch davon, dass zwischen den Naturwissenschaften und der Philosophie ein kooperatives Verbältnis bestehen soll, und bezeichnen diese Variante des Naturalismus daher als „kooperativen Naturalismus“ (Koppelberg 2000: 89). Zwischen dieser Kooperationsthese und der zuvor erläuterten Antifundierungsthese, die von nahezu allen Naturalisten geteilt wird und meiner Ansicht nach nicht so schnell aufgegeben werden sollte, besteht allerdings eine beachtliche Spannung: Denn insofern Kooperation als eine wechselseitige Beziehung verstanden wird, ist es mehr als seltsam, dass die Philosophie keinerlei Einfluss auf die Naturwissenschaften haben darf, wohl aber umgekehrt. Empirische Naturalisten fordern also meist keine wechselseitige Kooperation zwischen Naturwissenschaftlern und Philosophen, sondern sie fordern alle Philosophen dazu auf, den Apriorismus und die Lehnstuhlphilosophie aufzugeben und beim Philosophieren stets die Ergebnisse der naturwissenschaftlichen Forschung zu berücksichtigen.

Die Plausibilität des empirischen Naturalismus steht und fällt mit der Spezifiziererung der Rolle des empirischen Wissens für die Philosophie (Keil u. Schnädelbach 2000: 32-38). Was genau ist mit ,berücksichtigen' oder ,einbeziehen' gemeint? Klar ist, dass darunter mehr zu verstehen ist als ,nicht im Widerspruch stehen'. Unklar bleibt aber, ob empirisches Wissen immer, d. h. in Bezug 
auf alle philosophischen Fragestellungen, berücksichtigt werden muss und ob immer alle relevanten empirischen Erkenntnisse in die philosophische Argumentation einbezogen werden müssen oder nur ausgewählte. Wie bestimmt man überhaupt die Relevan₹ empirischer Erkenntnisse für bestimmte philosophische Fragestellungen und ist das nicht genau der Punkt, an dem der empirische Naturalist behaupten muss, dass empirisches Wissen immer (bzw. häufig) relevant ist? Erinnern wir uns an ein Ergebnis der bisherigen Erörterung: Der universale Anspruch ist dem Naturalismus eigen, zumindest insofern es sich um eine substantielle philosophische Position handeln soll. Dass es überhaupt Fragestellungen in der Philosophie gibt, für deren Beantwortung Philosophen einen (kurzen) Blick auf einige Erkenntnisse der Naturwissenschaftler werfen müssen, würde kaum jemand bezweifeln. Auch die Position des empirischen Naturalismus wird folglich erst durch ihren universalen Anspruch interessant.

\section{Empirischer Naturalismus:}

Die Beantwortung philosophischen Fragestellungen geschieht zwar unter Anwendung philosophischer (und nicht naturwissenschaftlicher) Methoden, aber in die Beantwortung aller Fragestellungen müssen empirische Erkenntnisse der Naturwissenschaften einbezogen werden.

\section{Was versteht man unter dem evolutionären Naturalismus in der Ethik?}

Wie wir in Abschnitt 2 gesehen haben, ist für den modernen Naturalisten typisch, dass er die Bedeutung der Naturwissenschaften im Vergleich zu und für andere Disziplinen (vor allem auch für die Philosophie) als sehr hoch einstuft. Betrachtet man den Spezialfall des evolutionären Naturalismus in der Ethik, so heißt das: Es geht um die Bedeutung einer sperifischen Naturwissenschaft, der biologischen Evolutionstheorie, für eine sperifische philosophische Disziplin, die Ethik. Wie ich bereits deutlich gemacht habe, kann die Spezifizierung der Position des evolutionären Naturalismus in der Ethik an dieser Stelle jedoch nicht stehen bleiben, ist doch nach wie vor unklar, was mit dem Ausdruck ,große Bedeutung' eigentlich genau gemeint ist. In Abschnitt 3 habe ich vier Naturalismusbegriffe und damit vier Weisen, ein Naturalist zu sein, unterschieden. Diese Begrifflichkeiten werde ich nun anwenden und untersuchen, welche in der Ethik diskutierten Positionen in welchem Sinne als (evolutionär) naturalistisch bezeichnet werden können. Ein wichtiger Schritt in dieser Analyse besteht darin, die Positionen danach zu differenzieren, in welcher der drei klassischen Bereiche der Ethik - der Metaethik, der normativen oder der deskriptiven Ethik - sie angesiedelt sind. Entsprechend dieser Dreiteilung werde ich im Folgenden drei Typen von Projekten einer evolutionären Naturalisierung der Ethik identifizieren und die Projekte in ihren Grundzügen erläutern.

\section{1 $\quad$...in der Metaethik?}

Die Metaethik wird auch als Disziplin zweiter Ordnung bezeichnet, weil es in ihr darum geht, die begrifflichen Grundlagen für die wissenschaftliche Auseinandersetzung mit dem Phänomen der Moral bereitzustellen. Insofern bildet sie die Grundlage sowohl für die normative als auch für die deskriptive Ethik (Düwell et al. 2006: 25-27). Standen zu Beginn nur sprachphilosophische Fragestellungen - wie die Frage nach der Bedeutung unserer moralischen Begriffe und die Frage nach der Bedeutung und dem Status moralischer Urteile - im Mittelpunkt (Moore [1903]), so wurde im Laufe der Jahrzehnte das ursprüngliche Feld metaethischer Diskussionen immer weiter vergrößert. Heutzutage werden in der Metaethik neben psychologischen und epistemologischen vorwiegend ontologische Fragestellungen diskutiert, was auf den Einfluss von John Mackie [1977] und 
Gilbert Harman (1977) zurückzuführen ist. Beispiele für solche ontologischen Fragen sind: Existieren in der Welt (unabhängig von subjektiven Leistungen) moralische Tatsachen? Gibt es moralische Eigenschaften und wenn ja, welchen Status besitzen sie?

Die Debatte um ontologische Fragen dieser Art ist auch der Ort, an dem die naturalistischen Positionen innerhalb der Metaethik zu finden sind. Ich möchte zwei Typen des metaethischen Naturalismus unterscheiden: eine naturalistische Version des Realismus und eine des Antirealismus. ${ }^{4}$ Metaethische Realisten behaupten, dass in der Welt moralische Tatsachen bzw. Eigenschaften existieren. Erinnern wir uns an die in Abschnitt 3 erläuterte Position des ontologischen Naturalismus, so scheint es zunächst widersprüchlich, den Realisten als Naturalisten zu bezeichnen, ist ein ontologischer Naturalist doch der Meinung, dass in der Welt außer natürlichen Entitäten keine Entitäten eines anderen Typs - und so auch keine moralischen Tatsachen oder Eigenschaften - existieren. Diese vermeintliche Spannung lässt sich durch eine zusätzliche These des Realisten aufheben, die seine Position naturalistisch werden lässt, die allerdings von verschiedenen Autoren unterschiedlich ausbuchstabiert wird: Moralische Tatsachen bzw. Eigenschaften seien nichts anderes als natürliche Tatsachen bzw. Eigenschaften, sie könnten auf sie reduziert werden bzw. sie seien durch sie konstituiert. Ist man der Meinung, dass sich moralische Tatsachen bzw. Eigenschaften auf natürlicher Tatsachen bzw. Eigenschaften eines ganz bestimmten Typs reduzieren lassen, nämlich auf evolutionäre Tatsachen bzw. auf Eigenschaften, die Gegenstand der Evolutionstheorie sind, so ist man ein evolutionärer naturalistischer Realist. Moralische Werte, für deren Existenz ein evolutionärer naturalistischer Realist argumentieren könnte, sind z. B. das individuelle Überleben, die erfolgreiche Aufzucht von eigenen Kindern, Neffen und Nichten sowie die Erhaltung der eigenen Stammlinie.

Im Gegensatz zum Realisten verneint der metaethische Antirealist explizit die Existenz moralischer Tatsachen bzw. Eigenschaften, weil eine solche Annahme für die Erklärung der moralischen Praxis nicht notwendig sei. ${ }^{5}$ Auch Antirealisten sind folglich ontologische Naturalisten, insofern sie behaupten, dass in der Welt nur natürliche Entitäten existieren. Die Position von Kitcher ist ein Beispiel für einen Antirealismus, der evolutionär naturalistisch ist. Kitcher argumentiert wie folgt: Menschen hätten im Laufe ihrer biologischen und kulturellen Evolution bestimmte psychologische Vermögen entwickelt, die sie auch in der heutigen Zeit dazu befähigten, bestimmte moralische Urteile zu fällen und bestimmte Normensysteme zu entwickeln. Betrachte man diese evolutionäre Entstehung der moralischen Praxis des Menschen, so finde man darin keinerlei Anzeichen für die Existenz moralischer Tatsachen in der Welt. Bestimmte Normen hätten sich nicht deshalb etabliert, weil sie moralische Tatsachen adäquat einfingen, die in der Welt existierten, sondern weil sie eine Verbesserung des sozialen Zusammenhaltes zwischen den Mitgliedern einer Gemeinschaft bewirkt hätten (Kitcher 2006a,b).

Wenden wir nun die in Abschnitt 3 eingeführten Begrifflichkeiten an und stellen uns die Frage, in welchem Sinne die metaethischen Realisten und Antirealisten Naturalisten sind. Wie schon deutlich geworden sein sollte, handelt es sich in beiden Fällen um einen Naturalismus im ontologischen Sinne. Sowohl die Realisten als auch die Antirealisten argumentieren - allerdings auf unterschiedliche Weise - für die ontologische These, dass ausschließlich natürliche Entitäten in

\footnotetext{
${ }^{4}$ In der Debatte ist dagegen üblich, mit dem Ausdruck ,Naturalismus' lediglich eine bestimmte Version der realistischen Position zu bezeichnen. Genau genommen gibt es aber in beiden Lager Naturalisten.

5 Der Antirealismus kann entweder mit einem Nonkognitivismus oder mit einer Irrtumstheorie verbunden werden. Nonkognitivisten vertreten die Ansicht, dass moralische Urteile nicht wahrheitsfähig sind bzw. dass in moralischen Urteilen keine Überzeugungen über moralische Tatsachen zum Ausdruck gebracht werden. Irrtumstheoretiker behaupten im Gegensatz dazu, dass moralische Urteile wahrheitsfähig sind bzw. dass sie Überzeugungen über moralische Tatsachen ausdrücken. Sie sind allerdings der Meinung, dass diese moralischen Urteile immer falsch sind, da in der Welt keine moralischen Tatsachen existieren.
} 
der Welt existieren. Beide Positionen sind evolutionär, weil evolutionsbiologische Erkenntnisse innerhalb der metaethischen Argumentation verwendet werden. Evolutionär naturalistische Realisten verwenden die Evolutionstheorie als Quelle der Ontologie, insofern sie aus ihr ableiten, auf welche natürlichen Tatsachen bzw. Eigenschaften moralische Tatsachen bzw. Eigenschaften reduziert werden können. Evolutionär naturalistische Antirealisten wie Kitcher ziehen bestimmte Forschungsergebnisse über die Evolution des Menschen als stütrende Belege für ihre metaethischen Thesen heran. Vor diesem Hintergrund stellt sich die Frage, ob die Naturalisten in der Metaethik nicht noch in einem zweiten Sinne Naturalisten sind. Schließlich setzen sowohl die Realisten als auch die Antirealisten (zumindest implizit) voraus, dass die Evolutionstheorie von großer Bedeutung dafür sein sollte, zu welchen Ergebnissen man innerhalb der Metaethik kommt. Meiner Analyse nach sind die metaethischen Naturalisten sowohl ontologische als auch empirische Naturalisten, aber in keinem Fall methodologische. Denn sowohl Realisten als auch Antirealisten betreiben nicht Metaethik unter Anwendung evolutionsbiologischer Methoden (wie z. B. der Methode der „historischen Rekonstruktion“ Mayr 2005: 52) und zeichnen diese evolutionsbiologischen Methoden als den einzigen verlässlichen Weg zur Wahrheit in der Metaethik aus. Stattdessen ziehen sie empirische Erkenntnisse der Evolutionstheoretiker dazu heran, um sie als Bestandteile in ihre metaethische Argumentation einzubauen. Die Forschungsergebnisse der Evolutionsbiologen in die philosophische Argumentation einzubeziehen, ist jedoch etwas ganz anderes, als Philosophie mit evolutionsbiologischen Methoden zu betreiben.

\section{2 $\quad$...in der normativen Ethik?}

Die Aufgabe der normativen Ethiker besteht in der Begründung und Rechtfertigung moralischer Normen. Gegenstand der normativen Ethik ist folglich die menschliche Moralität im normativen Sinne, d. h. die Menge von Normen, die aufgestellt und befolgt werden sollen. Die Menge der moralischen Normen, die de facto aufgestellt und befolgt werden, fällt dagegen in das Gebiet der deskriptiven Ethik (vgl. Abschnitt 3.3). Eine normativ ethische Theorie liefert eine Antwort auf die Frage, worin die richtige Moral besteht, d. h. welche Handlungsweisen moralisch erlaubt, welche verboten und welche moralisch indifferent sind (Düwell et al. 2006: 25, 61 f.)

Um die naturalistischen Positionen in der normativen Ethik werden sowohl die meisten als auch die hitzigsten Diskussionen innerhalb der Debatte um die evolutionäre Ethik geführt. Die Idee der evolutionären Naturalisten ist die Folgende: Der Mensch sei ein Produkt der biologischen Evolution. Aus der Perspektive der heutigen Forschung sei es plausibel, dass sich nicht nur viele der körperlichen Merkmale des Menschen, sondern auch (zumindest einige) seiner Verhaltensdispositionen in adaptiven Selektionsprozessen herausgebildet hätten. Warum sollte also nicht auch die menschliche Moralfähigkeit wesentlich durch die biologische Evolution geformt sein? Im Tierreich könnten doch auch Vorstufen der menschlichen Moralität - die so genannte „Proto-Moralität“ (Kitcher 2006a: 173) - beobachtet werden, die klarerweise das Ergebnis biologischer Evolution seien. Wie könne eine solche evolutionäre Bedingtheit der menschlichen Moral irrelevant für die Frage sein, welche moralischen Normen gelten sollten? Dieser letzte Schritt ist derjenige, den die Kritiker des evolutionären Naturalismus in der normativen Ethik nicht mitgehen. Sie betonen den Unterschied zwischen den empirischen Fakten einerseits und den moralischen Normen andererseits und weisen darauf hin, dass man - wie schon Hume gezeigt habe (Treatise: Buch III, Teil I) - nicht vom Sein auf das Sollen schließen könne. Aus der Tatsache, dass es im Lichte der biologischen Evolution beispielsweise vorteilhaft ist, seinen engsten Verwandten zu helfen (weil man dadurch indirekt die Weitergabe seiner eigenen Gene befördert), folge nicht, dass die moralische Norm, seinen engsten Verwandten zu helfen, auch gelten solle. Obwohl der Sein-Sollen-Schluss von einigen Befürwortern der evolutionären 
Ethik nicht als ein Fehlschluss angesehen wird (Wilson 1978) bzw. versucht wird, die Trennung zwischen Fakten und Normen aufzuheben (Voland 2004), sind sich die meisten Autoren darin einig, dass auf der Basis von empirischen Erkenntnissen über die Evolution des Menschen keine moralischen Normen gerechtfertigt werden können.

Gibt man dieses Projekt einer evolutionären Naturalisierung der normativen Ethik auf, so bleiben noch zwei weitere, weniger problematische Versuche übrig, für die Bedeutung der Evolutionstheorie für die normative Ethik zu argumentieren. Evolutionsbiologische Erkenntnisse können zum einen dafür verwendet werden, bestimmte Behauptungen über die Natur und den Ursprung der Lebewesen (z. B. die Auffassung, dass der Mensch von Gott durch einen speziellen Akt der Schöpfung geschaffen worden ist) als empirisch falsch zu erweisen. Insofern sich eine ethische Theorie auf eine solche empirisch falsche Annahme stützt, kann die Evolutionstheorie diesen Stütspfeiler der Theorie untergraben und - sofern keine weiteren Stützpfeiler vorhanden sind - den begründeten Status dieser ethischen Theorie in Frage stellen. William FitzPatrick spricht auch von der korrigierenden Funktion der Evolutionsbiologie innerhalb der normativen Ethik (FitzPatrick 2008: 20 f.). Diese Art der Naturalisierung der normativen Ethik ist unter den Disputanten zwar wenig kontrovers, allerdings in ihrem Anwendungsbereich auch sehr eingeschränkt.

Eine andere Möglichkeit der evolutionären Naturalisierung der normativen Ethik besteht darin, die Ergebnisse der Forschung über die Evolution des Menschen dazu heranzuziehen, die Grenzen der Lebbarkeit der Moral zu bestimmen. Setzt man voraus, dass innerhalb der normativen Ethik nur solche moralischen Normen rechtfertigbar sind, die von Menschen auch prinzipiell befolgt werden könnten (ganz nach dem Prinzip ,Sollen impliziert Können), dann könnten evolutionsbiologische Erkenntnisse zwar nicht die Grundlage für die Begründung von Normen bilden, sie können aber dabei den Rahmen abstecken, innerhalb dessen normative Ethiker moralische Normen rechtfertigen könnten. Evolutionsbiologen seien demnach zwar nicht in der Lage, die positive Frage zu beantworten, welche Normen gelten sollten, sie würden aber zur Beantwortung der negativen Frage beitragen, welche Normen nicht gelten sollten. Die Voraussetzung dieses Naturalisierungsprojektes, nämlich die Annahme, dass normative Ethik nicht idealistisch sein darf und der begrenzten Moralfähigkeit des Menschen Rechnung tragen muss, ist jedoch keineswegs unumstritten (z. B. Craemer-Ruegenberg 1993).

Die Frage, welches Verständnis von Naturalismus den soeben erläuterten drei Naturalisierungsversuchen der normativen Ethik zugrunde liegt, ist schnell beantwortet. Es handelt sich nicht um einen Naturalismus im ontologischen Sinne, weil Naturalisten innerhalb der normativen Ethik keine These darüber aufstellen, welche Entitäten in der Welt existieren. Insofern die Metaethik die Grundlage der normativen Ethik bildet, können ontologische Thesen zwar zu den Voraussetzungen einer naturalistischen normativ-ethischen Theorie gehören, diese ist aber selbst keine naturalistische Position im ontologischen Sinne. Es geht auch nicht um einen Naturalismus im methodologischen Sinne, denn die These der Naturalisten ist nicht, dass man normative Ethik mit evolutionsbiologischen Methoden betreiben soll, sondern dass empirisches Wissen in die philosophische Erörterung normativ-ethischer Fragestellungen mit einbezogen werden soll. Evolutionäre Naturalisten in der normativen Ethik sind also empirische Naturalisten, die fordern, dass Tatsachenbehauptungen über die Evolution des Menschen einen wesentlichen Teil der philosophischen Argumentation für eine ethische These bilden sollen - sei es nun im Rahmen der Rechtfertigung von Normen, der Argumentation gegen des begründeten Status einer ethischen Theorie oder der Bestimmung der Rechtfertigbarkeit von Normen. 
Angesichts der umfangreichen und nur schwer zu begegnenden Kritik, die den evolutionären Ethikern bei dem Versuch einer evolutionären Rechtfertigung moralischer Normen entgegenschlägt, beschränken sich viele von ihnen mittlerweile darauf, lediglich eine evolutionäre Erklärung der menschlichen Moralität geben zu wollen (Boniolo u. De Anna 2006, Joyce 2006, Sinnott-Armstrong 2008). Damit verlagern sie ihr Naturalisierungsprojekt von der normativen in die deskriptive Ethik, in der es nicht um die Begründung der moralischen Normen geht, die befolgt werden sollen, sondern um die Beschreibung und Erklärung derjenigen moralischen Normen, die in einer bestimmten Gemeinschaft faktisch anerkannt sind (Moralität im empirischen Sinne). Dabei geht es nicht nur um moralische Normen, sondern um die Erklärung der gesamten Vielfalt an de facto vorhandenen moralischen Phänomenen (moralische Urteile, Gefühle, Vermögen, Verhaltensweisen, Handlungen, Systeme etc.). Dass die biologische Evolution neben anderen phänotypischen Merkmalen des Menschen auch seine Fähigkeit zur Moralität geformt hat und insofern die Evolutionsbiologie nicht irrelevant dafür sein kann, die verschiedenen Elemente der heutigen moralischen Praxis des Menschen zu erklären, scheint unkontrovers. Strittig wird die These erst, wenn es um die Details geht.

Sehen wir uns die Details deshalb ein wenig genauer an: Die erste Frage, die sich für einen deskriptiven Ethiker stellt, ist die nach den Explananda: Welches sind die moralischen Phänomene, die es zu erklären gilt? Die Explananda sind auf zwei Ebenen angesiedelt: Zum einen geht es auf der Ebene der Individuen um die Erklärung derjenigen Verbaltensweisen (bzw. Handlungen) von Menschen, die als moralisch angesehen werden. Den moralischen Verhaltensweisen gehen häufig moralische Urteile voran. Seit der Kritik der evolutionären Psychologen an der Soziobiologie weiß man allerdings, dass menschliche Verhaltensmerkmale selbst nur schlecht einer evolutionären Erklärung zugänglich sind (Sterelny/Griffiths 1999). Die evolutionären Psychologen haben sich deshalb darauf verständigt, nicht das Verhalten selbst, sondern die dem Verhalten zugrunde liegenden psychologischen Mechanismen als die zu erklärenden Phänomene zu identifizieren. Dementsprechend vertreten die evolutionäre Naturalisten in der deskriptiven Ethik die folgende These: Das menschliche Moralverhalten und -urteilen lasse sich in dem Sinne evolutionär erklären, dass sich bestimmte kognitive bzw. emotionale Vermögen identifizieren ließen, die erstens Voraussetzungen für bzw. Einflussfaktoren auf die Bildung moralischer Urteile oder unmittelbar auf das moralische Verhalten seien, und die zweitens durch biologische Evolution entstanden seien, d. h. eine genetische Grundlage besäßen und das Ergebnis adaptiver Selektionsprozesse seien. Zum anderen sind die Explananda der deskriptiven Ethik auf der Ebene von moralischen Gemeinschaften angesiedelt. Auf dieser Ebene gilt es, die in einer Gemeinschaft anerkannten moralischen Normen und die in ihr etablierten Normensysteme evolutionär zu erklären. Ob die Evolutionsbiologie in der Lage ist, die heutigen Systeme moralischer Normen zu erklären, wird kontroverser diskutiert als im Falle des Moralverhaltens individueller Menschen (Kitcher 2006a,b, Ayala 2006, Parmigiani et al. 2006).

Für das Projekt einer evolutionären Naturalisierung der deskriptiven Ethik ist noch eine weitere Unterscheidung wichtig: Evolutionäre Naturalisten können zum einen behaupten, im Menschen existiere ein allgemeines Moralvermögen (z. B. ein Vermögen der normativen Urteilsbildung oder ein Sinn für Moralität), das evolutionär entstanden sei und Menschen dazu in die Lage versetze, überhaupt moralische Urteile zu fällen und sich von diesen leiten zu lassen, das aber den Inhalt der moralischen Urteile nicht beeinflusse. Zum anderen können sie die Existenz bereichssperifischer Moralvermögen annehmen, die durch biologische Evolution geformt seien und die Menschen dazu befähigten, unter bestimmten Bedingungen moralische Urteile mit einem bestimmten 
Inhalt zu fällen bzw. moralische Verhaltensweisen eines bestimmten Typs zu zeigen. Beide Thesen können unabhängig voneinander vertreten werden (FitzPatrick 2008: 6 f.).

Kommen wir nun zu der Frage, was ein evolutionärer Naturalist in der deskriptiven Ethik eigentlich genau behauptet. Evolutionäre Naturalisten schreiben der Evolutionsbiologie in zwei Hinsichten eine große Bedeutung für die Erklärung der menschlichen Moralität zu - sie behaupten, die Evolutionsbiologie könne einen großen Bereich der moralischen Urteile bzw. Verhaltensweisen des Menschen erklären und die Erklärungen hätten eine große explanatorische Kraft:

(1) Große Erklärungsreichweite:

Evolutionär entstandene Moralvermögen beeinflussen das Auftreten aller oder den Inhalt vieler moralischer Urteile bzw. Verhaltensweisen.

\section{(2) Große Erklärungskraft:}

Evolutionär entstandene Moralvermögen besitzen im Einzelfall im Vergleich zu anderen Faktoren einen großen Einfluss auf (a) das Auftreten moralischer Urteile bzw. Verhaltensweisen oder auf (b) den Inhalt moralischer Urteile bzw. den Typ moralischer Verhaltensweisen.

Je nachdem, ob ein evolutionärer Naturalist die Evolution eines allgemeinen Moralvermögens oder verschiedener bereichsspezifischer Moralvermögen annimmt, lässt sich leichter entweder für These (1) oder (2) argumentieren: Ein allgemeines Moralvermögen würde zwar alle moralischen Urteile bzw. Verhaltensweisen beeinflussen (Erklärungsreichweite sehr groß), aber der Einfluss wäre im Einzelfall nicht sehr groß, weil es nur das Auftreten, nicht aber den Inhalt der moralischen Urteile bzw. den Typ der Verhaltensweisen beeinflussen würde (Erklärungskraft nicht so groß). Bereichsspezifische Moralvermögen würden dagegen nur in einer höheren Anzahl viele moralischen Urteile bzw. Verhaltensweisen beeinflussen (Erklärungsreichweite nicht so groß), ihr Einfluss wäre aber im Einzelfall sehr groß, weil sie auch den Inhalt der moralischen Urteile bzw. den Typ der moralischen Verhaltensweisen beeinflussen würden (Erklärungskraft sehr groß).

Auf der Basis der bisherigen Forschungen scheint es unplausibel, anzunehmen, bei den moralischen Urteilen bzw. Verhaltensweisen des Menschen handle es sich um eine bomogene Menge, die auf der Basis weniger evolutionärer Vermögen erklärt werden können. Wahrscheinlicher ist, dass es eine Pluralität von Einflussfaktoren gibt, die nicht alle in den Gegenstandsbereich der Evolutionsbiologie fallen, und es daher noch andere Wissenschaften bedarf, um zu wirklich zufrieden stellenden Erklärungen der menschlichen Moralität zu gelangen. Dem evolutionären Naturalisten bleibt an dieser Stelle nichts anderes übrig, als darauf zu hoffen, dass sich seine Thesen sich als empirisch richtig erweisen werden. Die eigentliche Beantwortung der Fragen, welche Moralvermögen durch biologische Evolution entstanden sind und wie groß ihr Einfluss im Einzelfall ist, muss er den Evolutionsbiologen (sowie anderen Wissenschaftlern) überlassen.

Bleibt noch zu klären, welcher der in Abschnitt 3 eingeführten Naturalismusbegriffe innerhalb der deskriptiven Ethik seine Anwendung findet. Es scheint klar zu sein, dass es nicht um die ontologische Weise geht, ein Naturalist zu sein. Denn bei der oben erläuterten naturalistischen Position geht es nicht um die Frage, ob in der Welt nur natürliche Entitäten oder auch Entitäten eines anderen Typs existieren. Es handelt sich auch nicht um einen Naturalismus im empirischen Sinne, denn der Naturalist innerhalb der deskriptiven Ethik vertritt keine Auffassung darüber, wie man Philosophie betreiben sollte und dass man in die philosophische Argumentation evolutionsbiologisches Wissen einbeziehen sollte. Der Grund dafür ist - und das mag zuerst vielleicht überraschen -, dass es sich bei der Entwicklung der evolutionären Erklärungen 
moralischer Urteile bzw. Verhaltensweisen des Menschen gar nicht um Philosophie, sondern um Evolutionsbiologie handelt. Der Aufgabenbereich der Philosophen innerhalb der deskriptiven Ethik umfasst stattdessen die Klärung der Bedeutung und des Zusammenhangs zwischen zentralen Begriffen und die Reflexion darüber, welches die zu erklärenden Phänomene sind, welche Adäquatheitsbedingungen sich für Erklärungen moralischer Phänomene formulieren lassen und welches Verhältnis zwischen den Erklärungen unterschiedlicher Disziplinen besteht. Ein empirischer Naturalist müsste in Bezug auf diese Fragestellungen fordern, dass sie unter Berücksichtigung evolutionsbiologischer Erkenntnisse beantwortet werden sollten. Das ist allerdings weder eine plausible Forderung noch eine These, die der tatsächliche evolutionäre Naturalist in der deskriptiven Ethik vertritt. In den obigen beiden Thesen bringt er vielmehr die Zuversicht zum Ausdruck, dass es der Evolutionsbiologie in der Zukunft gelingen wird, für nahezu alle moralischen Urteile bzw. Verhaltensweisen des Menschen Erklärungen mit einer großen Erklärungskeraft zu liefern. Damit legt er sich jedoch auch nicht auf die beiden Thesen des starken methodologischen Naturalismus fest, die evolutionsbiologischen (bzw. die naturwissenschaftlichen) Methoden seien der einæige Weg, um zu einer adäquaten Erklärung aller moralischen Urteile bzw. Verhaltensweisen des Menschen zu gelangen. Er vertritt damit auch keinen methodologischen Naturalismus im schwachen Sinne, obwohl die Forderung, dass bei der Suche nach einer Erklärung der menschlichen Moralität zunächst ausschließlich evolutionsbiologische Methoden angewendet werden sollten, zu seiner Zuversichtsbekundung passen würde. Meine Antwort auf die Frage, in welchem Sinne hier von einem evolutionären Naturalismus die Rede ist, kann also nur lauten: Die Position des evolutionären Naturalisten in der deskriptiven Ethik geht über eine bloße Respekt- und Solidaritätsbekundung gegenüber der Evolutionsbiologie nicht wesentlich hinaus.

\section{4}

\section{Schlussbemerkung}

Fassen wir zusammen: Befürworter einer evolutionären Naturalisierung der Ethik behaupten, dass die Evolutionsbiologie von entscheidender Bedeutung für die Ethik ist bzw. sein sollte. Diese Behauptung konnte unter Anwendung der eingeführten Begrifflichkeiten und mit Blick auf die drei klassischen Bereiche der Ethik spezifiziert werden: Die in der Metaethik diskutierten Versionen des Naturalismus sind sowohl im ontologischen als auch im empirischen Sinne naturalistisch: Metaethische Naturalisten argumentieren für die ontologische These, dass ausschließlich natürliche Entitäten in der Welt existieren, und sie tun dies, indem sie empirische Forschungsergebnisse über die biologische Evolution des Menschen als Bestandteile in ihre metaethische Argumentation einbauen. Bei den Naturalisierungsversuchen der normativen Ethik handelt es sich ebenfalls um die Version des empirischen Naturalismus. Auch in diesem ethischen Gebiet ist die Forderung der Naturalisten nicht, dass die Ethik mit evolutionsbiologischen Methoden betrieben werden soll (methodologischer Naturalismus), sondern dass relevantes empirisches Wissen der Evolutionsbiologen in die philosophische Erörterung normativ-ethischer Fragestellungen mit einbezogen werden soll. Innerhalb der deskriptiven Ethik vertritt der Naturalist keine These darüber, wie man Ethik betreiben sollte (ob unter Anwendung evolutionsbiologischer Methoden oder unter Einbeziehung evolutionsbiologischer Forschungsergebnisse). Stattdessen besteht seine Position ausschließlich in der Zuversichtsbekundung, dass es der Evolutionsbiologie in der (nahen oder fernen) Zukunft gelingen wird, für nahezu alle moralischen Urteile bzw. Verhaltensweisen des Menschen Erklärungen mit einer großen Erklärungskraft zu liefern. Eine solche Position kann nicht als eine substantielle Version des Naturalismus bezeichnet werden. 


\section{Literaturverzeichnis}

Armstrong DM (1983) What Is a Law of Nature? Cambridge, Cambridge University Press

Ayala F (2006) Biology to Ethics: An Evolutionist's View of Human Nature. In: Boniolo, De Anna G (eds) Evolutionary Ethics and Contemporary Biology. Cambridge, Cambridge University Press, S. 141-158

Bayertz K (1993a) Der evolutionäre Naturalismus in der Ethik. In: Lütterfelds W (Hrsg): Evolutionäre Ethik zwischen Naturalismus und Idealismus: Beiträge zu einer modernen Theorie der Moral. Darmstadt, WTB, S. 141-165

Boniolo G, De Anna G (2006a) (eds) Evolutionary Ethics and Contemporary Biology. Cambridge, Cambridge University Press

Carrier M (2007) Wege der Wissenschaftsphilosophie im 20. Jabrbundert. In: Bartels A u. Stöckler M (Hrsg) Wissenschaftstheorie. Ein Studienbuch. Paderborn, mentis, S. 15-44

Cartwright N (1999) The Dappled World. A Study of the Boundaries of Science. Cambridge, Cambridge University Press

Craemer-Ruegenberg I (1993) „Evolutionäre Ethik“ und „Idealistische Ethik“. In: Lütterfelds W (Hrsg) Evolutionäre Ethik zwischen Naturalismus und Idealismus: Beiträge zu einer modernen Theorie der Moral. Darmstadt, WTB, S. 166-179

Düwell M, Hübenthal C u. Werner MH (2006) (Hrsg) Handbuch Etbik. 2., aktual. und erw. Aufl. Stuttgart, Metzler

Esfeld M (2007) Metaphysics of science between metaphysics and science. Grazer Philosophische Studien 74: $199-213$

Feldman R (2008) Naturalized Epistemology. Stanford Encyclopedia of Philosophy. Fall Edition (www.plato.stanford.edu/entries/epistemology-naturalized/)

Fine A (1996) The Shaky Game: Einstein, Realism, and the Quantum Theory. 2. Aufl. Chicago, University of Chicago Press

FitzPatrick W (2008) Morality and Evolutionary Biology. Stanford Encyclopedia of Philosophy. Winter Edition (www.plato.stanford.edu/entries/morality-biology/)

Forrest B (2000) Methodological Naturalism and Philosophical Naturalism: Clarifying the Connection. Philo 3(2): 7-29

Gesang B (2005) Normative Wissenschaftstheorie - Ein längst verstorbener Patient? In: Ders (Hrsg) Deskriptive oder normative Wissenschaftstheorie? Frankfurt, ontos, S. 7-30

Harman G (1977) The Nature of Morality. An Introduction to Ethics. Oxford, Oxford University Press

Hartmann D, Lange R (2000) Ist der erkenntnistheoretische Naturalismus gescheitert? In: Keil G, Schnädelbach H (Hrsg) Naturalismus. Philosophische Beiträge. Frankfurt/Main, Suhrkamp, S. 144-162

Hume D (1978 [1739/40]) A Treatise of Human Nature. Hrsg. von Selby-Bigge LA, Nidditch PH. 2. Ausg. Oxford, Oxford University Press (abgekürzt mit Treatise)

Joyce R (2006) The Evolution of Morality. Cambridge (u. a.), Bradford Book

Keil G, Schnädelbach H (2000) Naturalismus. In: Dies (Hrsg) Naturalismus. Philosophische Beiträge. Frankfurt/Main, Suhrkamp, 7-45

Kim J (2006) Philosophy of Mind. 2. Aufl. Boulder, Westview Press 
Kim J (1988) What is Naturalized Epistemology? In: Tomberlin JE (ed) Philosophical Perspectives 2. Asascadero, Ridgeview Publishing Co, S. 381-406

Kitcher P (1993) Vier Arten, die Ethik zu „biologisieren“. In: Bayertz K (Hrsg) Evolution und Ethik. Stuttgart, Reclam, S. 221-242

Kitcher P (2006a) Biology and Ethics. In: Copp D (ed) The Oxford Handbook of Ethical Theory. Oxford, Oxford University Press, S. 163-185

Kitcher P (2006b) Between Fragile Altruism and Morality. In: Boniolo G, De Anna G (eds) Evolutionary Ethics and Contemporary Biology. Cambridge, Cambridge University Press, S. 159-177

Koppelberg D (1996) Was macht eine Erkenntnistheorie naturalistisch? Journal for General Philosophy of Science 27(1): 71-90

Koppelberg D (2000) Was ist Naturalismus in der gegenwärtigen Philosophie? In: Keil G, Schnädelbach H (Hrsg) Naturalismus. Philosophische Beiträge. Frankfurt/Main, Suhrkamp, S. 68-91

Kornblith H (1994) Naturalism: Both Metaphysical and Epistemological. Midwest Studies in Philosophy 19: $39-52$

Kummer C (2009) Der Fall Darwin. Evolutionstheorie contra Schöpfungsglaube. München, Pattloch

Kurtz P (1998) Darwin Re-Crucified - Why Are So Many Afraid of Naturalism? Free Inquiry Magazine 18(2): 15-17

Ladyman J/Ross D (2007) (eds) Every Thing Must Go. Metaphysics Naturalized. Oxford, Oxford University Press

Mackie J (1990 [1977]) Ethics: Inventing Right and Wrong. New York, Penguin Books

Mayr E (2005) Konzepte der Biologie. Stuttgart, S. Hirzel

Maudlin T (2007) The Metaphysics within Physics. Oxford, Oxford University Press

Mittelstraß J (1984) Natur. In: Ders (Hrsg) Enayklopädie Philosophie und Wissenschaftstheorie. Bd. 2. Mannheim (u.a.), S. 961-964

Moore GE (2004 [1903]) Principia Ethica. Cambridge, Cambridge University Press

Papineau D (2009): Naturalism. Stanford Encyclopedia of Philosophy. Spring Edition. (www.plato.stanford.edu/entries/naturalism/)

Parmigiani S, De Anna G, Mainardi D u. Palanza P (2006) The Biology of Human Culture and Ethics: An Evolutionary Perspective. In: Boniolo G, De Anna G (eds) Evolutionary Ethics and Contemporary Biology. Cambridge, Cambridge University Press, S. 121-138

Quine WVO (1975) Naturalisierte Erkenntnistheorie. In: Ders Ontologische Relativität und andere Schriften. Stuttgart, Reclam

Quine WVO (1981) Theories and Things. Cambridge, Harvard University Press

Ritchie J (2008) Understanding Naturalism. Stocksfield, Acumen

Ruse M (2008) Creationism. In: Stanford Encyclopedia of Philosophy. Fall Edition. (www.plato.stanford.edu/entries/creationism/)

Sellars RW (1922) Evolutionary Naturalism. Chicago, Open Court

Sellars W (1956) Empiricism and the Philosophy of Mind. In: Feigl H/Scriven M (eds) The Foundations of Science and the Concepts of Psychoanalysis. Minneapolis, University of Minnesota Press, S. 127-196

Sinnott-Armstrong W (2008) (ed) Moral Psychology. Volume 1: The Evolution of Morality: Adaptations and Innateness. Cambridge (u. a.), Bradford Book 
Smart JJC (1959) Sensations and Brain Processes. The Philosophical Review 68(2): 141-156

Sterelny K, Griffiths P (1999) Sex and Death. An Introduction to Philosophy of Biology. Chicago (u. a.), The University of Chicago Press

Stich S (1993) Naturalizing Epistemology: Quine, Simon and the Prospects for Pragmatism. In: Hookway C, Peterson D (eds) Philosophy and Cognitive Science. Cambridge, Cambridge University Press, S. 1-17

Voland E (2004) Genese und Geltung - Das Legitimationsdilemma der Evolutionären Ethik und ein Vorschlag zu seiner Überwindung. Philosophia naturalis 41: 139-153

Voland E (2009) Evaluating the Evolutionary Status of Religiosity and Religiousness. In: ead, Schiefenhövel W (eds) The Biological Evolution of Religious Mind and Behavior. Berlin, Springer, S. $9-24$

Vollmer G (2000) Was ist Naturalismus? In: Keil G, Schnädelbach H (Hrsg) Naturalismus. Philosophische Beiträge. Frankfurt/Main, Suhrkamp, S. 46-67

Wilson EO (1975) Sociobiology. The New Synthesis. Cambridge (u. a.), Harvard University Press

Wilson EO (1978) On Human Nature. Cambridge (u. a.), Harvard University Press 